\title{
HIGH PRECISION ASTROMETRY OVER LARGE ANGULAR SCALES WITH CLOSURE CONSTRAINTS: THE TRIPLET $1803+784 / 1928+738 / 2007+777$
}

\author{
E. ROS ${ }^{1}$, J.M. MARCAIDE ${ }^{1}$, J.C. GUIRADO ${ }^{2}$, T.P. KRICHBAUM ${ }^{3}$, \\ R.A. PRESTON ${ }^{2}$, M.I. RATNER ${ }^{4}$, I.I. SHAPIRO ${ }^{4}$ AND A. WITZEL $^{3}$ \\ 1 Departamento de Astronomía, Universitat de València, Dr. \\ Moliner 50, E-46100 Burjassot (València), Spain \\ 2 Jet Propulsion Laboratory, California Institute of Technology, \\ 4800 Oak Grove Drive, Pasadena, California 91109 \\ ${ }^{3}$ Max-Planck Institut für Radioastronomie, Auf dem Hügel 69, \\ D-53121 Bonn, Germany \\ 4 Harvard-Smithsonian Center for Astrophysics, 60 Garden \\ St., Cambridge, Massachusetts 02138
}

The technique of differential astrometry using the phase-delay VLBI observable promises fractional precisions of $\sim 2 \times 10^{-9}$ in the determination of the separation of sources $5^{\circ}$ or $6^{\circ}$ apart on the sky (Guirado et al. 1995a; Lara et al. 1996). In our present research we seek further improvement in this technique through using triplets of radio sources, which provide a closure constraint in the determination of relative angular positions. This constraint not only eases the resolution of the phase-cycle ambiguities (a major problem in the least-squares approach to astrometry with phase delays), but it also strongly constrains the space of allowable parameter values.

The radio sources $1803+784,1928+738$, and $2007+777$, hereafter labelled A, B, and C, respectively, were cyclically observed on 20 November 1991 with a 10-antenna VLBI array. These sources are taken from the S5 radio survey (Kühr et al. 1981) and are part of an ongoing VLBI monitoring program (e.g. Witzel et al. 1988). We have phase-connected the data following a standard procedure (Shapiro et al. 1979, Guirado et al. 1995a, $1995 \mathrm{~b}$ ) for sets of data corresponding to the pairs of radio sources B-C, C-A, and A-B. For our present analysis, we used only data at $\lambda 3.6 \mathrm{~cm}$ from Bonn, 
Haystack, VLBA-PT, and VLBA-LA. After the standard phase-connection process we corrected the phase delays for the estimated contribution of the structure of the radio sources, but not for the ionospheric contribution. We have also demonstrated the possibility of estimating simultaneously the three angular separations in a "global" solution, but we have not yet used the full power of demanding zero sky-closure as a constraint in the weighted-least-squares analysis.

We define the sky-closure $\mathcal{C}_{\alpha \delta}$ of a triplet of radio sources as a "circular" sum of the estimated relative angular separations for the three pairs of radio sources: $\mathcal{C}_{\alpha \delta}=\left(\sum \Delta \alpha, \sum \Delta \delta\right)$, where $\Delta \alpha, \Delta \delta$ symbolize the differences in right ascensions and declinations for the various pairs of sources. In our case:

$$
\mathcal{C}_{\alpha \delta}=\left(\Delta \alpha_{(A-B)}+\Delta \alpha_{(B-C)}+\Delta \alpha_{(C-A)}, \Delta \delta_{(A-B)}+\Delta \delta_{(B-C)}+\Delta \delta_{(C-A)}\right)
$$

Preliminary analyses of the data yield the following angular separations (estimated independently for each pair of sources) and statistical standard errors:

$$
\begin{aligned}
& \left(\Delta \alpha_{(A-B)}, \Delta \delta_{(A-B)}\right)=\left(-1^{h} 27^{m} 02^{s} .81069 \pm 0^{8} .00002,4^{\circ} 30^{\prime} 02^{\prime \prime} .4481 \pm 0^{\prime \prime} .0001\right) \\
& \left(\Delta \alpha_{(B-C)}, \Delta \delta_{(B-C)}\right)=\left(-0^{h} 37^{m} 42^{s} .50316 \pm 0^{s} .00002,-3^{\circ} 54^{\prime} 41^{\prime \prime} .6773 \pm 0^{\prime \prime} .0001\right) \\
& \left(\Delta \alpha_{(C-A)}, \Delta \delta_{(C-A)}\right)=\left(2^{h} 04^{m} 45^{\circ} .31386 \pm 0^{s} .00002,-0^{\circ} 35^{\prime} 20^{\prime \prime} .7707 \pm 0^{\prime \prime} .0001\right)
\end{aligned}
$$

$\mathcal{C}_{\alpha \delta}$ should be consistent with zero for both coordinates. The above results yield:

$$
\mathcal{C}_{\alpha \delta}=\left(0^{h} 0^{m} 0^{s} .00001,0^{\circ} 0^{\prime} 0^{\prime \prime} .0001\right)
$$

The relative separation obtained for the pair $1928+738 / 2007+777$ at 3.6 $\mathrm{cm}$ is compatible with the preliminary results of the $3.6 / 13 \mathrm{~cm}$ observations from 1988 (Elósegui 1991) and with the final result of the $6 \mathrm{~cm}$ observations from 1985 (Guirado et al. 1995a). Minor differences between the solutions are possibly due to opacity effects between 3.6 and $6 \mathrm{~cm}$, and, perhaps also, to small changes in the reference features used in the maps.

\section{References}

Elósegui, P., 1991, Ph.D. thesis, Universidad de Granada.

Guirado, J.C. et al., $A \& A, 293,513,(1995 \mathrm{a})$

Guirado, J.C. et al., $A J$, (in the press), (1995b)

Kühr, H. et al., $A J, 86,854$, (1981)

Lara, L. et al., $A \& A$, (in the press), (1996)

Shapiro, I.I. et al., $A J, 84,1459$, (1979)

Witzel, A. et al., $A \& A, 206,245,(1988)$ 